Aim(s)/objectives The aim of this study was to explore experiences and strategies of married African-born women who are living in the United Kingdom in prevention of HIV.

Methods Eighteen in-depth Interviews were conducted with married African-born women who were aged between 25 to 55 years old in three Scottish cities: Aberdeen; Edinburgh; and, Glasgow.

Results Women's reports suggest a false sense of security amongst married women in regard to HIV prevention. Contrary to the daily exposure to the lived realities of HIV in Africa, HIV is rarely mentioned in media or discussed by health professionals. Condom use and asking husbands to get HIV tested was deemed unnecessary and therefore often neglected.

Discussion/conclusion Policies and interventions for HIV prevention amongst married African-born women should transcend multiple levels: individual-level; couple-level; and, structurallevel.

\section{P253 REGIONAL AUDIT OF TESTING CHILDREN OF HIV POSITIVE MOTHERS}

${ }^{1}$ Victoria McArdell*, ${ }^{2}$ Katrina Humphreys, ${ }^{2}$ Sangeetha Sundaram, ${ }^{2}$ Raj Patel, ${ }^{2}$ Selvavelu Samraj. 'University of Southampton, Southampton, UK; ${ }^{2}$ Solent NHS Trust, Southampton, UK

\subsection{6/sextrans-2015-052126.295}

Background In 2009, the "Don't forget the children" report recommended that all new HIV-positive patients attending adult HIV services should have any children identified, tested and the information clearly documented. In our clinic, HIV diagnosis in a child was delayed due to lack of a robust testing protocol despite regularly engaging with the mother for her care. We aimed to survey our clinic's testing practice before and after publication of this report to assess impact.

Method A retrospective case note review on all HIV positive women registered at the Solent adult HIV service. The population will be divided into 2 groups: (a) pre guidelines $(\mathrm{n}=81)$, and post guidelines $(\mathrm{n}=61)$. Details of children, their ages, country of residence, testing status, outcomes and timescales were recorded.

Results

\begin{tabular}{|c|c|c|}
\hline & $\begin{array}{l}\text { Pre-guidelines } \\
\text { (2000-2009) } \\
\mathrm{n}=81\end{array}$ & $\begin{array}{l}\text { Post-guidelines } \\
\text { (2010-2014) } \\
n=61\end{array}$ \\
\hline Number of children $<18$, UK resident, at risk & 36 & 33 \\
\hline $\begin{array}{l}\text { Number of children for whom HIV testing } \\
\text { was discussed and documented in maternal } \\
\text { notes }\end{array}$ & $22(61 \%)$ & $33(100 \%)$ \\
\hline Testing initiated by HIV service & 10 & 15 \\
\hline Time scale for children to be tested (range) & 3 months - 9 years & 3 months -3 years \\
\hline
\end{tabular}

Conclusion Testing of children at risk of HIV has significantly improved in our service since the publication of "Don't forget the Children". However this audit identified some children who continue to remain untested or status unconfirmed. We have implemented a robust protocol to chase up outcomes of children tested outside of HIV service and to proactively negotiate testing when parents initially decline consent. Since January 2012, Southampton has been integrated with 3 other clinics to form Solent Sexual Health Service. We plan to extend this retrospective audit to include HIV positive women attending 3 other clinics, which may result in identification and testing of more children at risk.

\section{P254 SAFEGUARDING CHILDREN IN SEXUAL HEALTH SERVICES - A GROWING CONCERN}

Christine Donohue, Nicola Fearnley, Sophie Brady*. Bradford Teaching Hospitals NHS Foundation Trust, Bradford, West Yorkshire, UK

\subsection{6/sextrans-2015-052126.296}

Background Additional focus on child sexual exploitation (CSE) and high profile safeguarding cases within the media has impacted on workload within sexual health services. Our trust has established pathways for sharing information about the most vulnerable children in the form of named nurse (for safeguarding children) notifications (NNN). These facilitate the triangulation of information and senior review of cases. Following integration in 2011 we have emphasised the need for all clinical staff working across different sites to recognise children at risk and notify cases.

Aim To quantify the NNN made from our integrated service as a measure of safeguarding children workload.

Methods Numbers of safeguarding referrals in the form of NNN initiated by our service over 3 years were obtained from the NNN database.

Results

\begin{tabular}{llllll}
\hline Year & January-March & April-June & July-September & October-December & Total \\
\hline 2012 & 0 & 0 & 2 & 1 & 3 \\
2013 & 3 & 4 & 6 & 7 & 20 \\
2014 & 11 & 19 & 18 & 23 & 71 \\
\hline
\end{tabular}

10 database entries were undated: 5 closed in 2012; 5 in 2013.

Discussion The workload in managing children at risk has increased as demonstrated by the large rise in NNN. It is important that the additional workload falling upon teams is recognised and particularly the disproportionate burden falling upon health advisors who may be supporting the young people in addition to advising colleagues. The marked increase may have resulted from community staff gaining more experience in recognising the signs of children in need. Further training, supervision and the use of a standardised proforma across all sites may also have contributed. 